\title{
BOUNDARY BEHAVIOR OF SUPERHARMONIC FUNCTIONS SATISFYING NONLINEAR INEQUALITIES IN A PLANAR SMOOTH DOMAIN
}

\author{
KENTARO HIRATA \\ (Received 9 November 2007; accepted 22 December 2008) \\ Communicated by A. M. Hassell
}

\begin{abstract}
This paper presents a sharp boundary growth estimate for all positive superharmonic functions $u$ in a smooth domain $\Omega$ in $\mathbb{R}^{2}$ satisfying the nonlinear inequality

$$
-\Delta u(x) \leq c \delta_{\Omega}(x)^{-\alpha} u(x)^{p} \quad \text { for all } x \in \Omega,
$$

where $c>0, \alpha \in \mathbb{R}$ and $p>0$, and $\delta_{\Omega}(x)$ stands for the distance from a point $x$ to the boundary of $\Omega$. A result is applied to show the existence of nontangential limits of such superharmonic functions.

2000 Mathematics subject classification: primary 31A20; secondary 31A05, 35J60.

Keywords and phrases: superharmonic function, boundary growth, nontangential limit, nonlinear elliptic equation.
\end{abstract}

\section{Introduction}

Many elliptic equations involving nonlinear terms have been studied widely from the viewpoints of not only differential equations but also (probabilistic) potential theory. See $[2,4,10-12]$ and references therein. In this paper, we present a sharp boundary growth estimate for positive superharmonic functions satisfying a certain nonlinear inequality in a planar smooth domain. Furthermore, we apply the result to show the existence of nontangential limits of such superharmonic functions. Let us start with the definition of superharmonic functions. By $D(x, r)$, we denote the open disk of center $x$ and radius $r$ in $\mathbb{R}^{2}$. Let $\Omega$ be a domain in $\mathbb{R}^{2}$. A lower semicontinuous function $u: \Omega \rightarrow(-\infty,+\infty]$, where $u \neq \equiv+\infty$, is called superharmonic on $\Omega$ if it satisfies the mean value inequality

$$
u(x) \geq \frac{1}{\pi r^{2}} \int_{D(x, r)} u(y) d y,
$$

This work was partially supported by Grant-in-Aid for Young Scientists (B) (No. 19740062), Japan Society for the Promotion of Science.

(C) 2009 Australian Mathematical Publishing Association Inc. 1446-7887/2009 \$16.00 
whenever the closure of $D(x, r)$ is contained in $\Omega$. Let $\Delta$ be the Laplacian on $\mathbb{R}^{2}$. It is well known that if $u$ is superharmonic on $\Omega$, then there exists a unique (Radon) measure $\mu_{u}$ on $\Omega$ such that

$$
\int_{\Omega} \phi(x) d \mu_{u}(x)=-\int_{\Omega} u(x) \Delta \phi(x) d x \quad \text { for all } \phi \in C_{0}^{\infty}(\Omega),
$$

where $C_{0}^{\infty}(\Omega)$ is the collection of all infinitely differentiable functions vanishing outside a compact set in $\Omega$. The measure $\mu_{u}$ is called the Riesz measure associated with $u$. If $\mu_{u}$ is absolutely continuous with respect to Lebesgue measure and $d \mu_{u}(x)=$ $f_{u}(x) d x$ where $f_{u}$ is a nonnegative locally integrable function on $\Omega$, then we call $f_{u}$ the Riesz function associated with $u$. If $u \in C^{2}(\Omega)$, then $f_{u}=-\Delta u$.

Throughout the paper, we suppose that $\Omega$ is a bounded $C^{1,1}$-domain in $\mathbb{R}^{2}$. By $\delta_{\Omega}(x)$ we denote the distance from a point $x$ to the boundary $\partial \Omega$ of $\Omega$. The symbol $A$ stands for an absolute positive constant whose value is unimportant and may change from line to line. We discuss positive superharmonic functions $u$ on $\Omega$ having an associated Riesz function $f_{u}$ and satisfying the nonlinear inequality

$$
f_{u}(x) \leq c \delta_{\Omega}(x)^{-\alpha} u(x)^{p} \quad \text { for almost every } x \in \Omega,
$$

where $c>0, \alpha \in \mathbb{R}$ and $p>0$. Our results are as follows.

THEOREM 1.1. Let $c>0$ and $p>0$. Suppose that

$$
\alpha \leq 3-p \text {. }
$$

Let $u$ be a positive superharmonic function on $\Omega$ having an associated Riesz function $f_{u}$ which satisfies (1.1). Then there exists a constant A depending only on $u, c, \alpha, p$ and $\Omega$ such that

$$
\frac{\delta_{\Omega}(x)}{A} \leq u(x) \leq \frac{A}{\delta_{\Omega}(x)} \text { for all } x \in \Omega .
$$

Actually, the lower bound estimate in (1.2) is valid for all positive superharmonic functions (see Lemma 3.2). Thus, the interesting object is the relation between the boundary growth estimate and the ranges of $p$ and $\alpha$. The following theorem shows that the bound $\alpha \leq 3-p$ is sharp for the upper bound estimate in (1.2). For $\xi \in \partial \Omega$ and $\theta>0$, let

$$
\Gamma_{\theta}(\xi)=\left\{x \in \Omega:|x-\xi|<(1+\theta) \delta_{\Omega}(x)\right\} .
$$

THEOREM 1.2. Let $c>0$ and $p>0$. Suppose that

$$
\alpha>3-p \text {. }
$$

Let $\xi \in \partial \Omega$. Then there exists a positive $C^{2}$-function $u$ on $\Omega$ satisfying

$$
0 \leq-\Delta u(x) \leq c \delta_{\Omega}(x)^{-\alpha} u(x)^{p} \quad \text { for all } x \in \Omega
$$

such that

$$
\limsup _{\Gamma_{\theta}(\xi) \ni x \rightarrow \xi} \delta_{\Omega}(x) u(x)=+\infty
$$

for any $\theta>0$. In particular, $u$ does not satisfy the upper bound estimate in (1.2). 
Higher dimensional results analogous with Theorems 1.1 and 1.2 are presented in another paper [5]. The above results are proved using similar ideas given there. However, the proof of Theorem 1.1 is much simpler than the higher dimensional case, because of the higher-order integrability of the logarithmic kernel. In contrast, the proof of Theorem 1.2 requires a careful choice of sequences of balls and numbers. Furthermore, combining Theorem 1.1 and the earlier result due to Arsove and Huber, we will obtain a result concerning the existence of nontangential limits in the next section.

\section{Application to nontangential limits}

It is well known that every positive superharmonic function in the unit disk has radial limits almost everywhere on the boundary (see Littlewood [7]). However, nontangential limits do not necessarily exist. The existence of nontangential limits was proved by Arsove and Huber [1] (see also references therein).

THEOREM A. Let u be a positive superharmonic function on the unit disk D having an associated Riesz function $f_{u}$. Suppose that there exists a constant A such that

$$
f_{u}(x) \leq \frac{A}{(1-|x|)^{2}} \quad \text { for almost every } x \in D .
$$

Then $u$ has nontangential limits almost everywhere on $\partial D$.

Now, we suppose that $p>0$ and $\alpha \leq 2-p$. Let $u$ be a positive superharmonic function on the unit disk $D$ having an associated Riesz function $f_{u}$ which satisfies

$$
f_{u}(x) \leq c(1-|x|)^{-\alpha} u(x)^{p} \quad \text { for almost every } x \in D .
$$

Then it follows from Theorem 1.1 that

$$
f_{u}(x) \leq A \frac{u(x)^{p}}{(1-|x|)^{\alpha}} \leq \frac{A}{(1-|x|)^{\alpha+p}} \leq \frac{A}{(1-|x|)^{2}} \quad \text { for almost every } x \in D .
$$

Therefore Theorem A implies that $u$ has nontangential limits almost everywhere on $\partial D$. Thus we obtain the following result.

Corollary 2.1. Let $c>0$ and $p>0$. Suppose that $\alpha \leq 2-p$. Let $u$ be a positive superharmonic function on the unit disk $D$ having an associated Riesz function $f_{u}$ which satisfies (2.1). Then u has nontangential limits almost everywhere on $\partial D$.

\section{Proof of Theorem 1.1}

For two positive functions $f$ and $g$, we write $f \approx g$ if there exists a constant $A>1$ such that $A^{-1} f \leq g \leq A f$. The constant $A$ will be called the constant of comparison. 
Let $G$ and $K$ denote the Green's function for $\Omega$ and the Martin kernel of $\Omega$ with pole at $\xi \in \partial \Omega$, respectively. It is known that

$$
G(x, y) \approx \log \left(1+\frac{\delta_{\Omega}(x) \delta_{\Omega}(y)}{|x-y|^{2}}\right) \text { for all } x, y \in \Omega,
$$

where the constant of comparison depends only on $\Omega$ (see [3]). Let $x_{0} \in \Omega$ be fixed and let $\left\{y_{j}\right\}$ be a sequence in $\Omega$ converging to $\xi \in \partial \Omega$. Since the Martin boundary of $\Omega$ coincides with the Euclidean boundary (see [6]), the ratio $G\left(x, y_{j}\right) / G\left(x_{0}, y_{j}\right)$ converges to $K(x, \xi)$. Therefore we obtain

$$
K(x, \xi) \approx \frac{\delta_{\Omega}(x)}{|x-\xi|^{2}} \quad \text { for all } x \in \Omega,
$$

where the constant of comparison depends only on $\Omega$.

LEMMA 3.1. If h is a positive harmonic function on $\Omega$, then there exists a constant $A$ depending only on $h$ and $\Omega$ such that

$$
\frac{\delta_{\Omega}(x)}{A} \leq h(x) \leq \frac{A}{\delta_{\Omega}(x)} \text { for all } x \in \Omega .
$$

PROOF. By the Martin representation theorem,

$$
h(x)=\int_{\partial \Omega} K(x, \xi) d v(\xi),
$$

where $v$ is a measure on $\partial \Omega$ such that $v(\partial \Omega)=h\left(x_{0}\right)>0$. Hence (3.2) yields

$$
\frac{\delta_{\Omega}(x)}{A} h\left(x_{0}\right) \leq h(x) \leq \frac{A}{\delta_{\Omega}(x)} h\left(x_{0}\right),
$$

and so the lemma follows.

LEMMA 3.2. If $u$ is a positive superharmonic function on $\Omega$, then there exists a constant $A$ depending only on $u$ and $\Omega$ such that

$$
u(x) \geq \frac{1}{A} \delta_{\Omega}(x) \text { for all } x \in \Omega .
$$

ProOF. Let $\mu_{u}$ be the Riesz measure associated with $u$. By the Riesz decomposition theorem,

$$
u(x)=h(x)+\int_{\Omega} G(x, y) d \mu_{u}(y) \text { for all } x \in \Omega,
$$

where $h$ is a nonnegative harmonic function on $\Omega$. If $\mu_{u}(\Omega)=0$, then $u=h$. Therefore we obtain (3.3) from Lemma 3.1 in this case. If $\mu_{u}(\Omega)>0$, then we find $r>0$ such that $\mu_{u}(E)>0$, where $E=\left\{x \in \Omega \mid \delta_{\Omega}(x) \geq r\right\}$. It follows from (3.1) that

$$
u(x) \geq \int_{E} G(x, y) d \mu_{u}(y) \geq \frac{\delta_{\Omega}(x)}{A} \mu_{u}(E) \quad \text { whenever } \delta_{\Omega}(x)<\frac{r}{2} .
$$

Also, $u$ has a positive minimum on $\left\{x \in \Omega \mid \delta_{\Omega}(x) \geq 2^{-1} r\right\}$ by the lower semicontinuity of $u$. Hence (3.3) follows. 
In the rest of this section, we suppose that $u$ is a positive superharmonic function on $\Omega$ having an associated Riesz function $f_{u}$ which satisfies (1.1). Then, by the Riesz decomposition theorem,

$$
u(x)=h(x)+\int_{\Omega} G(x, y) f_{u}(y) d y \quad \text { for all } x \in \Omega,
$$

where $h$ is the greatest harmonic minorant of $u$ on $\Omega$. Note that $h$ is nonnegative.

LEMma 3.3. There exists a constant A depending only on $u$ and $\Omega$ such that

$$
\int_{\Omega} \delta_{\Omega}(y) f_{u}(y) d y \leq A .
$$

PROOF. Since $u$ is finite almost everywhere on $\Omega$, we can find a point $x_{1} \in \Omega$ with $u\left(x_{1}\right)<\infty$. Observe from (3.1) that $G\left(x_{1}, y\right) \geq A^{-1} \delta_{\Omega}(y)$ for all $y \in \Omega$. Since $f_{u} \geq 0$, the conclusion follows from (3.4).

LEMMA 3.4. Let $x \in \Omega$ and let $4^{-1} \delta_{\Omega}(x) \leq \rho \leq 2^{-1} \delta_{\Omega}(x)$. Then, for all $z \in$ $D\left(x, 2^{-1} \rho\right)$,

$$
u(z) \leq \frac{A_{1}}{\delta_{\Omega}(x)}+A_{1} \int_{D(x, \rho)} f_{u}(y) \log \frac{3 \delta_{\Omega}(x)}{|z-y|} d y,
$$

where $A_{1}$ is a positive constant depending only on $u$ and $\Omega$.

PROOF. Let $x \in \Omega$ and $z \in D\left(x, 2^{-1} \rho\right)$. By (3.1),

$$
G(z, y) \leq A \frac{\delta_{\Omega}(y)}{\delta_{\Omega}(x)} \quad \text { for all } y \in \Omega \backslash D(x, \rho) .
$$

Therefore, the superharmonicity $f_{u} \geq 0$ and Lemma 3.3 give

$$
\int_{\Omega \backslash D(x, \rho)} G(z, y) f_{u}(y) d y \leq \frac{A}{\delta_{\Omega}(x)} .
$$

Also, by (3.1),

$$
G(z, y) \leq A \log \frac{3 \delta_{\Omega}(x)}{|z-y|} \quad \text { for all } y \in D(x, \rho) .
$$

Hence the conclusion follows from (3.4) and Lemma 3.1.

PROOF OF THEOREM 1.1. The lower bound estimate in (1.2) has been already proved in Lemma 3.2. We show the upper bound estimate. Let $x \in \Omega$. By Lemma 3.3,

$$
\delta_{\Omega}(x) \int_{D\left(x, 2^{-1} \delta_{\Omega}(x)\right)} f_{u}(y) d y \leq A .
$$


Let $r=\delta_{\Omega}(x)$ and let $\psi_{x}(\zeta)=r^{3} f_{u}(x+r \zeta)$. For simplicity, we write $D(r)=$ $D(0, r)$. By the change of variables $z=x+r \eta$ and $y=x+r \zeta$,

$$
\int_{D\left(2^{-1}\right)} \psi_{x}(\zeta) d \zeta \leq A
$$

and from Lemma 3.4

$$
\begin{aligned}
& r u(x) \leq A_{1}+A_{1} \int_{D\left(4^{-1}\right)} \psi_{x}(\zeta) \log \frac{3}{|\zeta|} d \zeta, \\
& r u(x+r \eta) \leq \Psi_{x}(\eta) \text { for all } \eta \in D\left(4^{-1}\right)
\end{aligned}
$$

where

$$
\Psi_{x}(\eta)=A_{1}+A_{1} \int_{D\left(2^{-1}\right)} \psi_{x}(\zeta) \log \frac{3}{|\eta-\zeta|} d \zeta .
$$

Suppose that $p>0$ and $\alpha \leq 3-p$. Let $q>\max \{1, p\}$ and $s=q / p>1$. By (3.6) and the Hölder inequality,

$$
r u(x) \leq A_{1}+A\left(\int_{D\left(4^{-1}\right)} \psi_{x}(\zeta)^{s} d \zeta\right)^{1 / s}
$$

Therefore, in order to show the upper bound estimate in (1.2), it suffices to prove that there exists a constant $A$ independent of $x$ such that

$$
\int_{D\left(4^{-1}\right)} \psi_{x}(\zeta)^{s} d \zeta \leq A
$$

Using the inequality $(a+b)^{t} \leq 2^{t}\left(a^{t}+b^{t}\right)$ for $a, b, t \geq 0$,

$$
\begin{aligned}
& \left(\int_{D\left(2^{-1}\right)} \Psi_{x}(\eta)^{q} d \eta\right)^{1 / q} \\
& \quad \leq A+A\left(\int_{D\left(2^{-1}\right)}\left(\int_{D\left(2^{-1}\right)} \psi_{x}(\zeta) \log \frac{3}{|\eta-\zeta|} d \zeta\right)^{q} d \eta\right)^{1 / q} .
\end{aligned}
$$

By the Minkowski inequality, the right hand side is bounded from above by

$$
A+A \int_{D\left(2^{-1}\right)}\left(\int_{D\left(2^{-1}\right)}\left(\log \frac{3}{|\eta-\zeta|}\right)^{q} d \eta\right)^{1 / q} \psi_{x}(\zeta) d \zeta
$$

Therefore

$$
\left(\int_{D\left(2^{-1}\right)} \Psi_{x}(\eta)^{q} d \eta\right)^{1 / q} \leq A+A \int_{D\left(2^{-1}\right)} \psi_{x}(\zeta) d \zeta .
$$

Since $2^{-1} r \leq \delta_{\Omega}(x+r \eta) \leq 2 r$ for all $\eta \in D\left(2^{-1}\right)$ and $r \leq \operatorname{diam} \Omega$, it follows from (1.1), (3.7) and $\alpha \leq 3-p$ that

$$
0 \leq \psi_{x}(\eta)=r^{3} f_{u}(x+r \eta) \leq c r^{3} \delta_{\Omega}(x+r \eta)^{-\alpha} u(x+r \eta)^{p} \leq A \Psi_{x}(\eta)^{p},
$$


for almost every $\eta \in D\left(4^{-1}\right)$. This and (3.9) give

$$
\left(\int_{D\left(4^{-1}\right)} \psi_{x}(\zeta)^{s} d \zeta\right)^{1 / q} \leq A+A \int_{D\left(2^{-1}\right)} \psi_{x}(\zeta) d \zeta
$$

because $s=q / p$. Hence (3.5) yields (3.8). This completes the proof.

\section{Proof of Theorem 1.2}

Proof of Theorem 1.2. Suppose that $p>0$ and $\alpha>3-p$. Let

$$
\lambda=p+\alpha \quad \text { and } \quad \gamma=\frac{\lambda-1}{2} .
$$

Then $\gamma>1$ and

$$
\lambda-2 \gamma=1
$$

Let $\xi \in \partial \Omega$. Since $\Omega$ is a $C^{1,1}$-domain, there is a disk $D(z, r)$ contained in $\Omega$ such that $\xi \in \partial D(z, r)$. Without loss of generality, we may assume that $\xi=(0,0)$, $z=(10,0)$ and $r=10$. For each $j \in \mathbb{N}$, let $x_{j}=\left(e^{-j^{3}+3}, 0\right)$ and $r_{j}=e^{-\gamma j^{3}}$. Then $D\left(x_{j}, 8 r_{j}\right) \subset \Omega$ and $D\left(x_{j}, 2 r_{j}\right) \cap D\left(x_{k}, 2 r_{k}\right)=\emptyset$ if $j \neq k$. Let $A_{2}$ be a constant to be determined below and let $f_{j}$ be a nonnegative smooth function on $\Omega$ such that

$$
f_{j} \leq A_{2} \frac{e^{\lambda j^{3}}}{j^{2}} \quad \text { on } \Omega \quad \text { and } \quad f_{j}= \begin{cases}A_{2} \frac{e^{\lambda j^{3}}}{j^{2}} & \text { on } D\left(x_{j}, r_{j}\right), \\ 0 & \text { on } \Omega \backslash D\left(x_{j}, 2 r_{j}\right)\end{cases}
$$

Let $A_{3}$ be the constant of comparison in (3.1) and let $j_{0}$ be a natural number such that

$$
\frac{c}{e^{3 \alpha}}\left(\frac{2 \pi A_{2}(\gamma-1)}{A_{3}}\right)^{p} \frac{j_{0}^{p+2}}{A_{2}} \geq 1
$$

Define $f=\sum_{j=j_{0}}^{\infty} f_{j}$. Then, by (4.1),

$$
\begin{aligned}
\int_{\Omega} \delta_{\Omega}(y) f(y) d y & =\sum_{j=j_{0}}^{\infty} \int_{D\left(x_{j}, 2 r_{j}\right)} \delta_{\Omega}(y) f_{j}(y) d y \\
& \leq \sum_{j=j_{0}}^{\infty} 2 \delta_{\Omega}\left(x_{j}\right) A_{2} \frac{e^{\lambda j^{3}}}{j^{2}} \pi\left(2 r_{j}\right)^{2} \\
& =8 e^{3} \pi A_{2} \sum_{j=j_{0}}^{\infty} \frac{e^{(-1+\lambda-2 \gamma) j^{3}}}{j^{2}}<\infty
\end{aligned}
$$


Hence $u:=\int_{\Omega} G(\cdot, y) f(y) d y$ is well defined on $\Omega$. Since $f$ is locally Hölder continuous on $\Omega$, it follows from [9, Theorem 6.6] that $u \in C^{2}(\Omega)$ and $u$ satisfies $-\Delta u=f$ in $\Omega$. For the moment, let $x \in \partial D\left(x_{j}, 2 r_{j}\right)$. By (3.1),

$$
\begin{aligned}
G\left(x, x_{j}\right) & \geq \frac{1}{A_{3}} \log \frac{\delta_{\Omega}(x) \delta_{\Omega}\left(x_{j}\right)}{\left|x-x_{j}\right|^{2}} \geq \frac{1}{A_{3}} \log \frac{2^{-1} \delta_{\Omega}\left(x_{j}\right)^{2}}{\left(2 r_{j}\right)^{2}} \\
& =\frac{1}{A_{3}} \log \frac{e^{6}}{8} e^{2(\gamma-1) j^{3}} \geq \frac{2(\gamma-1)}{A_{3}} j^{3} .
\end{aligned}
$$

Therefore the mean value equality and (4.1) give

$$
\begin{aligned}
u(x) & \geq \int_{D\left(x_{j}, r_{j}\right)} G(x, y) f_{j}(y) d y=A_{2} \frac{e^{\lambda j^{3}}}{j^{2}} \int_{D\left(x_{j}, r_{j}\right)} G(x, y) d y \\
& =A_{2} \frac{e^{\lambda j^{3}}}{j^{2}} \pi r_{j}^{2} G\left(x, x_{j}\right) \geq \frac{2 \pi A_{2}(\gamma-1)}{A_{3}} j e^{j^{3}} .
\end{aligned}
$$

By the minimum principle,

$$
u(x) \geq \frac{2 \pi A_{2}(\gamma-1)}{A_{3}} j e^{j^{3}} \quad \text { for all } x \in D\left(x_{j}, 2 r_{j}\right) .
$$

Hence

$$
u\left(x_{j}\right) \geq \frac{2 e^{3} \pi A_{2}(\gamma-1)}{A_{3}} j \delta_{\Omega}\left(x_{j}\right)^{-1},
$$

and so

$$
\lim _{j \rightarrow+\infty} \delta_{\Omega}\left(x_{j}\right) u\left(x_{j}\right)=+\infty .
$$

Finally, we show that $-\Delta u \leq c \delta_{\Omega}(x)^{-\alpha} u^{p}$ in $\Omega$. If $x \notin \bigcup_{j \geq j_{0}} D\left(x_{j}, 2 r_{j}\right)$, then

$$
c \delta_{\Omega}(x)^{-\alpha} u(x)^{p} \geq 0=f(x)=-\Delta u(x) .
$$

Let $x \in D\left(x_{j}, 2 r_{j}\right)$, where $j \geq j_{0}$. Then, by (4.2) and (4.3),

$$
\begin{aligned}
c \delta_{\Omega}(x)^{-\alpha} u(x)^{p} & \geq \frac{c}{e^{3 \alpha}}\left(\frac{2 \pi A_{2}(\gamma-1)}{A_{3}}\right)^{p} j^{p} e^{(p+\alpha) j^{3}} \\
& \geq f_{j}(x)=f(x)=-\Delta u(x) .
\end{aligned}
$$

This completes the proof.

\section{Acknowledgement}

The author is grateful to the editor for several valuable comments. 


\section{References}

[1] M. Arsove and A. Huber, 'On the existence of non-tangential limits of subharmonic functions', J. London Math. Soc. 42 (1967), 125-132.

[2] Z. Q. Chen, R. J. Williams and Z. Zhao, 'On the existence of positive solutions of semilinear elliptic equations with Dirichlet boundary conditions', Math. Ann. 298(3) (1994), 543-556.

[3] K. L. Chung and Z. Zhao, From Brownian Motion to Schrödinger's Equation, Grundlehren der math. Wissenschaften, 312 (Springer, Berlin, 1995).

[4] K. Hirata, 'Sharp estimates for the Green function, 3G inequalities, and nonlinear Schrödinger problems in uniform cones', J. Anal. Math. 99 (2006), 309-332.

[5] _ - 'The boundary growth of superharmonic functions and positive solutions of nonlinear elliptic equations', Math. Ann. 340(3) (2008), 625-645.

[6] R. A. Hunt and R. L. Wheeden, 'Positive harmonic functions on Lipschitz domains', Trans. Amer. Math. Soc. 147 (1970), 507-527.

[7] J. E. Littlewood, 'On functions subharmonic in a circle (II)', Proc. London Math. Soc. (2) 28 (1928), 383-394.

[8] H. Mâagli and L. Mâatoug, 'Singular solutions of a nonlinear equation in bounded domains of $\mathbf{R}^{2}$, J. Math. Anal. Appl. 270(1) (2002), 230-246.

[9] S. C. Port and C. J. Stone, Brownian Motion and Classical Potential Theory (Academic Press, New York, 1978).

[10] U. Ufuktepe and Z. Zhao, 'Positive solutions of nonlinear elliptic equations in the Euclidean plane', Proc. Amer. Math. Soc. 126(12) (1998), 3681-3692.

[11] Q. S. Zhang and Z. Zhao, 'Singular solutions of semilinear elliptic and parabolic equations', Math. Ann. 310(4) (1998), 777-794.

[12] Z. Zhao, 'On the existence of positive solutions of nonlinear elliptic equations-a probabilistic potential theory approach', Duke Math. J. 69(2) (1993), 247-258.

KENTARO HIRATA, Faculty of Education and Human Studies, Akita University, Akita 010-8502, Japan

e-mail: hirata@math.akita-u.ac.jp 